Journal of Algebra Combinatorics Discrete Structures and Applications

\title{
Weak isometries of Hamming spaces
}

Research Article

\section{Ryan Bruner, Stefaan De Winter}

\begin{abstract}
Consider any permutation of the elements of a (finite) metric space that preserves a specific distance $p$. When is such a permutation automatically an isometry of the metric space? In this note we study this problem for the Hamming spaces $H(n, q)$ both from a linear algebraic and combinatorial point of view. We obtain some sufficient conditions for the question to have an affirmative answer, as well as pose some interesting open problems.
\end{abstract}

2010 MSC: 05C12, 05C50

Keywords: Hamming space, Weak isometry, Eigenvalue collapsing

\section{Introduction}

Distance preserving permutations of metric spaces, better known as isometries, play a prominent role throughout mathematics. Their weaker counterparts, not surprisingly called weak isometries, are permutations of a metric space that preserve only a prescribed subset of distances. In certain cases it is known that a weak isometry necessarily has to be an isometry. A famous example is the Beckman-Quarles Theorem that states that every mapping on the real Euclidean space $\mathbb{R}^{n}, n>1$, that preserves distance 1 has to be an isometry [2].

Beckman-Quarles type results for certain finite metric spaces related to geometries and buildings were obtained by Govaert and Van Maldeghem in [6, 7], and by Abramenko and Van Maldeghem in [1]. In most cases the conclusion is that weak isometries are automatically isometries, however some interesting counter examples do exist. In particular, there exist permutations of the point set of the so-called split Cayley hexagon that preserve distance 6 between points, but do not arise from automorphisms of the hexagon, and hence do not give rise to isometries of the related metric space (see Section 3 of [7]).

An important class of finite metric spaces are provided by the distance regular graphs, and an important subclass of these are the Hamming spaces $H(n, q)$. These are the spaces $\{0,1,2, \ldots, q-1\}^{n}$ equipped with the Hamming distance. In [8] necessary and sufficient conditions were derived for a weak isometry of $H(n, 2)$ to be an isometry. In [5] all weak isometries of $H(n, 2)$ were classified. In this note we will focus on weak isometries of $H(n, q), q>2$.

Ryan Bruner, Stefaan De Winter (Corresponding Author); Department of Mathematical Sciences, Michigan Technological University, USA (email: rwbruner@mtu.edu, sgdewint@mtu.edu). 


\section{Definitions and preliminary observations}

Let $\Gamma$ be any finite connected simple graph without loops. Then $\Gamma$ can in an obvious way be viewed as a metric space in which the maximal distance equals the diameter, say $d$, of the graph. Now let $\phi$ be any permutation of the vertex set of $\Gamma$ that maps vertices at distance $p$ to vertices at distance $p$, for some specific $1 \leq p \leq d$. We will call such permutation a $p$-isometry of $\Gamma$. One can easily generalize this definition to that of a $P$-isometry, where $P \subset\{1,2, \ldots, d\}$. These are exactly the permutations of $\Gamma$ that preserve all distances $p \in P$. We are interested in the following question: "Under which conditions is a p-isometry automatically an isometry (= automorphism) of $\Gamma$ ?". In general this question will be very hard to answer, and if one does not assume some extra conditions on $\Gamma$, there might exist many $p$-isometries that are not isometries. Assume for example that the distance $p$-graph of $\Gamma$ (this is the graph in which two vertices are adjacent iff they are at distance $p$ in $\Gamma$ ) is not connected, and that one of the connected components, say $C_{1}$, has a non-trivial automorphism, say $\sigma$. Then one could consider the map $\tau$ on $\Gamma$ defined by $\tau(v)=\sigma(v)$ for $v \in C_{1}$, and $\tau(v)=v$ otherwise. In many cases $\tau$ will then not induce an isometry of $\Gamma$. An example of this idea is the construction of so-called even isometries of the Boolean cube (see Theorem 3 in [5]). It is however not true that all proper $p$-isometries arise in this way (see for example Theorem 6 and 8 in [5]). Nevertheless, the following restrictions on $\Gamma$ seem to be quite natural for our problem:

- the distance $p$-graph of $\Gamma$ is connected;

- $\Gamma$ admits a primitive automorphism group.

Even under these conditions it seems to be hopeless to find a general answer to our problem, and restricting to specific interesting classes of graphs seems to be the natural thing to do (and is what has been done so far, see for example the references mentioned in Sections 1). A very interesting class of graphs in this respect is the class of primitive distance regular graphs, as these graphs naturally fulfill both conditions mentioned above (for all $p$ ). However, even this class seems to be too general to allow a uniform answer to our question as there are both examples where a $p$-isometry is automatically an isometry, and cases where the group of $p$-isometries is larger than the automorphism group of the original graph. A small example in the latter class is given by the 3-isometries of the Odd graph $O_{4}$ on 35 vertices. In this case the group of 3-isometries is 8 times larger than the automorphism group of the original graph (this example was provided to the authors by J. Koolen and J. Williford, and can easily be confirmed with Sage or GAP). In this paper we will focus on the class of Hamming graphs $H(n, q)$ with $q>2$.

\section{Linear algebraic approach}

When trying to show that every $p$-isometry of $H(n, q), q>2$, is an isometry, a first natural approach is to make use of the Bose-Mesner algebra. The advantage of this approach is that it can be applied to any distance regular graph. For details on distance regular graphs and the Bose-Mesner algebra we refer to [3]. So for now, let $\Gamma$ be a distance regular graph of diameter $n$ with intersection numbers $a_{i}, b_{i}$ and $c_{i}, i=0,1, \ldots, n$. Furthermore, let $\Gamma_{i}$ denote the distance $i$-graph of $\Gamma$, that is, the graph on the same vertices as $\Gamma$ in which two vertices are adjacent if they are at distance $i$ in $\Gamma$. Let $A_{i}$ denote the adjacency matrix of $\Gamma_{i}$. Now assume $\phi$ is a $p$-isometry of $\Gamma$. This is equivalent with saying that $\phi$ is an automorphism of $\Gamma_{p}$, and hence, if we let $P$ denote the permutation matrix corresponding to $\phi$, with $P A_{p} P^{-1}=A_{p}$.

Theorem 3.1. Every $p$-isometry of the distance regular graph $\Gamma$ of diameter $n$ is a graph automorphism (of $\Gamma$ ) if the degree of the minimal polynomial of $A_{p}$ equals $n+1$.

Proof. Let $\phi$ be a $p$-isometry of $\Gamma$. As above, let $A_{i}$ denote the adjacency matrix of $\Gamma_{i}$, set $A=A_{1}$, and let $P$ denote the permutation matrix corresponding to $\phi$. We want to show that $P A P^{-1}=A$ under 
the conditions of the theorem. A natural way to do this will be to prove that $A$ can be written as a polynomial in $A_{p}$. It is well known, see for example [3], that

$$
\begin{gathered}
A_{0}=I, A_{1}=A \\
A A_{i}=c_{i+1} A_{i+1}+a_{i} A_{i}+b_{i-1} A_{i-1}(i=0, \ldots, n) \\
A_{0}+A_{1}+\ldots+A_{n}=J
\end{gathered}
$$

where $J$ is the all one matrix, $A_{-1}=A_{n+1}=0$, and $b_{-1}$ and $c_{n+1}$ are unspecified. From this it follows right away that $A_{p}$ is a polynomial of degree $p$ in $A$. However, we want to obtain the converse.

Recall that $\mathcal{A}=\left\{\alpha_{0} I+\alpha_{1} A+\ldots+\alpha_{n} A^{n}: \alpha_{i} \in \mathbb{C}\right\}$, the so-called Bose-Mesner algebra, is a commutative algebra. Obviously $A_{p}$ and all its powers belong to $\mathcal{A}$. We can write

$$
\begin{gathered}
A_{p}=\alpha_{01} I+\alpha_{11} A+\alpha_{21} A^{2}+\ldots+\alpha_{n 1} A^{n} \\
A_{p}^{2}=\alpha_{02} I+\alpha_{12} A+\alpha_{22} A^{2}+\ldots+\alpha_{n 2} A^{n} \\
\vdots \\
A_{p}^{n}=\alpha_{0 n} I+\alpha_{1 n} A+\alpha_{2 n} A^{2}+\ldots+\alpha_{n n} A^{n}
\end{gathered}
$$

As we want to write $A$ as a polynomial in $A_{p}$, we may see the above as a system of $n$ linear equations in the "unknowns" $A, A^{2}, \ldots, A^{n}$. It now is obvious we will be able to solve for $A$ (in terms of $A_{p}, A_{p}^{2}, \ldots, A_{p}^{n}$ ) if and only if $A_{p}, A_{p}^{2}, \ldots, A_{p}^{n}$ are linearly independent, that is, if and only if the degree of the minimal polynomial of $A_{p}$ is at least $n+1$. On the other hand, as $A_{p} \in \mathcal{A}$ the degree of this minimal polynomial is at most $n+1$.

The most obvious way to guarantee that the minimal degree of $A_{p}$ will be $n+1$ is that $A_{p}$ has $n+1$ distinct eigenvalues. We call the phenomenon of $A_{p}$ having fewer than $n+1$ distinct eigenvalues eigenvalue collapsing.

From here on let $\Gamma$ be the Hamming graph $H(n, q)$. It is well known that in this case the intersection numbers are given by $a_{i}=i(q-2), b_{i}=(n-i)(q-1)$, and $c_{i}=i$, for $i=0,1,2, \ldots, n$. Let $f_{i}$ denote the polynomial of degree $i$ such that $A_{i}=f_{i}(A)$. From the above it follows that the $f_{i}$ are recursively defined by

$$
\begin{gathered}
f_{-1}(x)=0, f_{0}(x)=1, f_{1}(x)=x \\
c_{i+1} f_{i+1}(x)=\left(x-a_{i}\right) f_{i}(x)-b_{i-1} f_{i-1}(x)(i=0, \ldots, n) .
\end{gathered}
$$

We know that the $n+1$ eigenvalues of $A$ are $\lambda_{i}=(q-1) n-q i$ for $i=0,1, \ldots, n$. Hence the eigenvalues of $A_{p}$ are

$$
\gamma_{i}=f_{p}\left(\lambda_{i}\right) \text { for } i=0,1, \ldots, n \text {. }
$$

If all $\gamma_{i}$ are distinct, then indeed by the above every $p$-isometry would be an isometry.

A straightforward check in Mathematica yielded the results presented in Tables 1 through 4 for $q=3,4,5$ and 6 . In these tables a value of "True" indicates that $A_{p}$ has $n+1$ distinct eigenvalues, 
whereas a value of "False" indicates that eigenvalue collapsing occured. We choose to present the tables for $q=3,4,5$ and 6 as these represent two primes, a prime power and a composite number. Our values of $n$ and $p$ run up to 12 , although one could easily compute many more. However, when we did so we did not uncover any interesting patterns. These results seem to indicate that in "most" cases $A_{p}$ has indeed $n+1$ distinct eigenvalues, but that eigenvalue collapsing occurs for seemingly random triples $(q, n, p)$. At this point we were unable to derive any meaningful theoretical conclusions on when eigenvalue collapsing for $H(n, q)$ will occur. The only exception is in the case $p=n$ where the recent results of Brouwer and Fiol [4] imply that no eigenvalue collapsing will occur. In their paper Brouwer and Fiol study eigenvalue collapsing in the case where $p$ equals the diameter for general distance regular graphs. In the next section we will discuss the $p=n$ case for $H(n, q)$ in a combinatorial way.

At this point one may wonder whether eigenvalue collapsing is equivalent with the existence of $p$-isometries that are not isometries. However, the next section will show this is not the case.

Table 1. $q=3$

\begin{tabular}{|c|c|c|c|c|c|c|c|c|c|c|c|}
\hline $\mathrm{q}=3$ & $p=2$ & 3 & 4 & 5 & 6 & 7 & 8 & 9 & 10 & 11 & 12 \\
\hline$n=3$ & True & True & & & & & & & & & \\
\hline 4 & False & False & True & & & & & & & & \\
\hline 5 & True & False & True & True & & & & & & & \\
\hline 6 & True & True & True & True & True & & & & & & \\
\hline 7 & False & False & True & False & False & True & & & & & \\
\hline 8 & True & True & True & True & True & True & True & & & & \\
\hline 9 & True & True & True & True & True & True & True & True & & & \\
\hline 10 & False & False & False & False & False & False & False & False & True & & \\
\hline 11 & True & False & True & True & False & True & False & False & True & True & \\
\hline 12 & True & True & True & True & True & True & True & True & True & True & True \\
\hline
\end{tabular}

Table 2. $\quad q=4$

\begin{tabular}{|c|c|c|c|c|c|c|c|c|c|c|c|}
\hline $\mathrm{q}=4$ & $p=2$ & 3 & 4 & 5 & 6 & 7 & 8 & 9 & 10 & 11 & 12 \\
\hline$n=3$ & False & True & & & & & & & & & \\
\hline 4 & True & True & True & & & & & & & & \\
\hline 5 & False & False & False & True & & & & & & & \\
\hline 6 & True & True & True & True & True & & & & & & \\
\hline 7 & False & True & False & True & False & True & & & & & \\
\hline 8 & True & True & True & True & True & True & True & & & & \\
\hline 9 & False & False & True & False & True & False & False & True & & & \\
\hline 10 & True & True & True & True & True & True & True & True & True & & \\
\hline 11 & False & True & False & True & False & True & False & True & False & True & \\
\hline 12 & True & True & True & True & True & True & True & True & True & True & True \\
\hline
\end{tabular}

Table 3. $q=5$

\begin{tabular}{|c|c|c|c|c|c|c|c|c|c|c|c|}
\hline $\mathrm{q}=5$ & $p=2$ & 3 & 4 & 5 & 6 & 7 & 8 & 9 & 10 & 11 & 12 \\
\hline$n=3$ & True & True & & & & & & & & & \\
\hline 4 & True & True & True & & & & & & & & \\
\hline 5 & True & True & True & True & & & & & & & \\
\hline 6 & False & False & True & False & True & & & & & & \\
\hline 7 & True & True & True & True & True & True & & & & & \\
\hline 8 & True & True & True & True & True & True & True & & & & \\
\hline 9 & True & True & True & True & True & True & True & True & & & \\
\hline 10 & True & True & True & True & True & True & True & True & True & & \\
\hline 11 & False & False & True & False & True & True & True & False & False & True & \\
\hline 12 & True & True & True & True & True & True & True & True & True & True & True \\
\hline
\end{tabular}


Table 4. $q=6$

\begin{tabular}{|c|c|c|c|c|c|c|c|c|c|c|c|}
\hline $\mathrm{q}=6$ & $p=2$ & 3 & 4 & 5 & 6 & 7 & 8 & 9 & 10 & 11 & 12 \\
\hline$n=3$ & True & True & & & & & & & & & \\
\hline 4 & False & True & True & & & & & & & & \\
\hline 5 & True & False & True & True & & & & & & & \\
\hline 6 & True & True & True & True & True & & & & & & \\
\hline 7 & False & False & True & True & False & True & & & & & \\
\hline 8 & True & True & True & True & True & True & True & & & & \\
\hline 9 & True & True & True & True & True & True & True & True & & & \\
\hline 10 & False & False & False & False & True & False & False & True & True & & \\
\hline 11 & True & False & False & True & False & True & True & True & True & True & \\
\hline 12 & True & True & True & True & True & True & True & True & True & True & True \\
\hline
\end{tabular}

\section{Combinatorial approach}

In this section we will present a proof that shows that in at least half of all possible cases every $p$-isometry of $H(n, q), q>2$ is an isometry. Our main result is the following.

Theorem 4.1. Let $\phi$ be a p-isometry of $H(n, q), q>2$. If $2 p<n$ or $p=n$ then $\phi$ is an isometry of $H(n, q)$.

We will proceed through a series of lemmas, but first need a few definitions. We will call the elements of $H(n, q)$ words. The weight of a word is simply its number of non-zero positions, and the support of a word is the set of nonzero positions in that word. The layer $L_{k}$ of weight $k$ of $H(n, q)$ is the set of all words of weight $k$ in $H(n, q)$. For $a<b$, the cloud $C(a, b)$ of $H(n, q)$ is the union of layers $\cup_{i=a}^{b} L_{i}$. We will denote the Hamming distance between two words $a$ and $b$ by $d(a, b)$. We will let 0 denote the unique word of weight zero. Finally, addition and subtraction in $H(n, q)$ are defined component wise modulo $q$. Throughout this section we always assume $q>2$.

Lemma 4.2. Let $\phi$ be a p-isometry of $H(n, q)$ fixing 0 and let $2 p<n$. Then, for positive integers $k$, the layers $L_{k p}, k p \leq n$, and the clouds $C((k-1) p+1, k p-1), k p \leq n$, are preserved set wise.

Proof. We start by noticing that only words of weight $p$ are at distance $p$ from 0 . Let $w$ be in $L_{p}$. Then

$$
p=d(0, w)=d(\phi(0), \phi(w))=d(0, \phi(w)) .
$$

Thus $\phi(w) \in L_{p}$, and $L_{p}$ is preserved as a set under $\phi$. Then $C(1, p-1) \cup C(p+1,2 p-1) \cup L_{2 p}$ are the only remaining words of $H(n, q)$ that are at distance $p$ from some word in $L_{p}$. So $C(1, p-1) \cup C(p+1$, $2 p-1) \cup L_{2 p}$ is preserved as a set under $\phi$. We know $C(2 p+1, n)$ is not empty since $n>2 p$. We also know $C(2 p+1, n)$ must be preserved set wise under $\phi$ as its complement is preserved. Because words in $C(p+1,2 p-1)$ and $L_{2 p}$ are at distance $p$ from some words in $C(2 p+1, n)$ while words in $C(1, p-1)$ are not, we see that $C(1, p-1)$ is preserved set wise as well as $C(p+1,2 p-1) \cup L_{2 p}$. Next notice that words in $L_{2 p}$ can never be at distance $p$ from words in $C(1, p-1)$ while every word in $C(p+1,2 p-1)$ is. Therefore both $C(p+1,2 p-1)$ and $L_{2 p}$ are preserved as sets.

The result now easily follows by induction.

Lemma 4.3. Let $2 p<n$. Two words of weight $p$ have disjoint support if and only if there exists a unique word of weight $2 p$ at distance $p$ from both words.

Proof. This is straightforward.

Lemma 4.4. Let $\phi$ be a p-isometry of $H(n, q)$ fixing 0 and let $2 p<n$. Then $\phi$ maps words of weight $p$ with disjoint support to words of weight $p$ with disjoint support. 
Proof. Let $\alpha$ and $\beta$ be the two words of weight $p$ with disjoint support. Let $\gamma$ be the unique word of weight $2 p$ at distance $p$ from both $\alpha$ and $\beta$. By Lemma $4.2 \phi$ maps $\alpha$ and $\beta$ to words of weight $p$ and $\gamma$ to a word of weight $2 p$. Furthermore, as $\phi$ is a $p$-isometry $\phi(\gamma)$ must be the unique word of weight $2 p$ at distance $p$ from $\phi(\alpha)$ and $\phi(\beta)$. Hence, by the previous lemma, $\phi(\alpha)$ and $\phi(\beta)$ have disjoint support.

Lemma 4.5. Assume $2 p<n$. A word $w$ in $C(1, p-1) \cup C(p+1,2 p-1)$ has even weight if and only if there are two words $x_{1}$ and $x_{2}$ of weight $p$ with disjoint support such that both $x_{1}$ and $x_{2}$ are at distance $p$ from $w$.

Proof. We first show that for a word $w$ of even weight in the given clouds there are two words $x_{1}$ and $x_{2}$ satisfying the conditions of the lemma. Let $w$ be a word of weight $2 k$ in $C(1, p-1) \cup C(p+1,2 p-1)$. Then we can construct two words $x_{1}$ and $x_{2}$ as desired using the simple construction shown below:

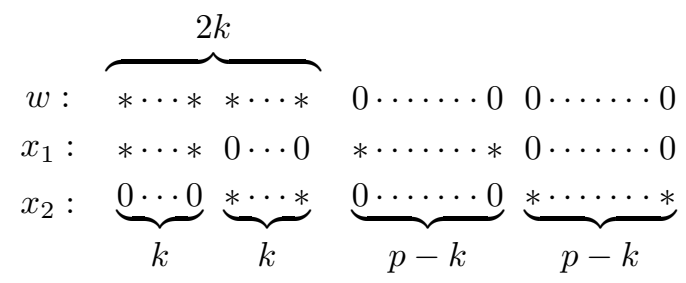

Next let $w$ be a word of weight $k$ in $C(1, p-1) \cup C(p+1,2 p-1)$ admitting two words $x_{1}$ and $x_{2}$ satisfying the conditions of the lemma. Let $a$ (respectively $a^{\prime}$ ) be the number of positions in the support of $w$ that are not in the support of $x_{1}$ (respectively $x_{2}$ ). Let $b$ (respectively $b^{\prime}$ ) be the number of positions where the support of $w$ and $x_{1}$ (respectively $x_{2}$ ) overlap but have different entries. Let $c$ (respectively $c^{\prime}$ ) be the number of positions where the support of $w$ and $x_{1}$ (respectively $x_{2}$ ) overlap and have equal entries. Finally let $d$ (respectively $d^{\prime}$ ) be the number of positions in the support of $x_{1}$ (respectively $x_{2}$ ) that are not in the support of $w$. The following pictographic representation of this situation allows to deduce a few key relationships:

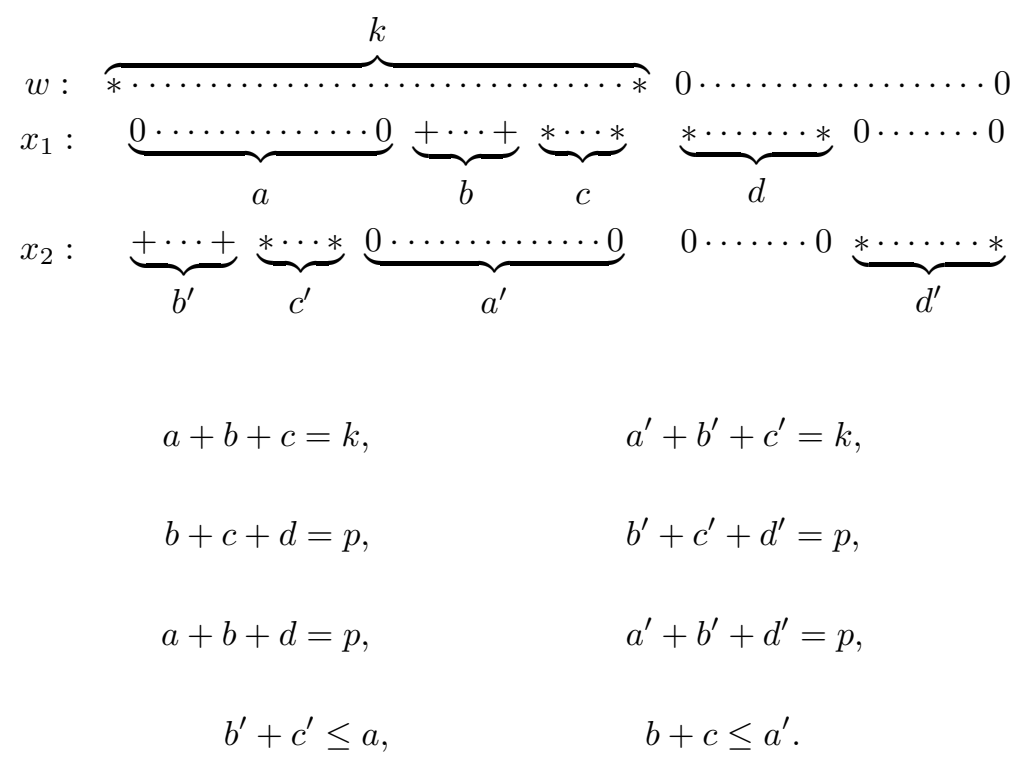

From $b+c+d=p=a+b+d$ we deduce that $a=c$, and similarly $a^{\prime}=c^{\prime}$. Then

$$
a+b=b+c \leq a^{\prime} \leq a^{\prime}+b^{\prime}=b^{\prime}+c^{\prime} \leq a .
$$

It follows that $b=0$. Hence $k=a+b+c=2 a$, and $w$ has even weight. 
Lemma 4.6. Let $2 p<n$, and let $\phi$ be a p-isometry of $H(n, q)$ fixing 0 . Then words of even (respectively odd) weight in $C(1, p-1)$ and $C(p+1,2 p-1)$ are mapped to words of even (respectively odd) weight in $C(1, p-1)$ and $C(p+1,2 p-1)$.

Proof. This follows immediately by combining Lemmas 4.2, 4.4 and 4.5.

Lemma 4.7. Let $2 p<n$, and let $\phi$ be a p-isometry of $H(n, q)$ fixing 0 . Then $\phi$ preserves the layer $L_{1}$.

Proof. First assume $p$ is odd. Set $p=2 k+1$. Notice that the only words at distance $p$ from a word of weight 1 in $C(p+1,2 p-1)$ are words of weight $p+1=2 k+2$. So all words at distance $p$ from a word of weight 1 in $C(p+1,2 p-1)$ have even weight. Now let us look at words in $C(2, p-1)$. All these words are at distance $p$ from both words of even weight and words of odd weight in $C(p+1,2 p-1)$. By Lemma 4.6 the parity of the words under consideration must be preserved by $\phi$. It follows that layer $L_{1}$ is stabilized by $\phi$.

In the same way, interchanging the roles of even and odd, we see that when $p$ is even, layer $L_{1}$ is preserved.

Lemma 4.8. Let $p=n$, and let $\phi$ be a $p$-isometry of $H(n, q)$ fixing 0 . Then $\phi$ preserves the layer $L_{1}$.

Proof. Let $w$ be a word of weight $x$. Then the number of words of weight $n$ at distance $n$ from $w$ equals $f(x)=(q-2)^{x}(q-1)^{n-x}$. So the number of words of weight $n$ at distance $n$ from a word of weight 1 equals $(q-2)^{1}(q-1)^{n-1}$. As $f(x)$ is a strictly decreasing function it follows that words of weight 1 are the only words that have $(q-2)^{1}(q-1)^{n-1}$ words of weight $n$ at distance $n$. Now we know that $n$-isometries that fix 0 preserve $L_{n}$. Hence, if there are $k$ words of weight $n$ at distance $n$ from a given word $w$, there should also be $k$ words of weight $n$ at distance $n$ from $\phi(w)$. It follows that $\phi$ stabilizes layer $L_{1}$.

We are now ready to prove Theorem 4.1.

Proof. Let $a$ and $c$ be words such that $d(a, c)=1$. Let $\phi(a)=b$. Then we construct the $p$-isometry $\psi:=\tau_{-b} \circ \phi \circ \tau_{a}$ where $\tau_{-b}$ and $\tau_{a}$ are isometries defined by $\tau_{a}(w)=w+a$ and $\tau_{-b}(w)=w-b$ for all words $w$. We note

$$
\psi(0)=\tau_{-b} \circ \phi \circ \tau_{a}(0)=\tau_{-b} \circ \phi(a)=\tau_{-b}(b)=0 .
$$

So $\psi$ is a $p$-isometry that fixes 0 . We obtain

$$
\begin{aligned}
& d(\phi(a), \phi(c)) \\
= & d\left(\tau_{-b} \circ \phi(a), \tau_{-b} \circ \phi(c)\right) \\
= & d\left(\tau_{-b} \circ \phi \circ \tau_{a}(0), \tau_{-b} \circ \phi \circ \tau_{a}(c-a)\right) \\
= & d(\psi(0), \psi(c-a)) \\
= & d(0, \psi(c-a))
\end{aligned}
$$

Now $d(c, a)=1$ implies that $d(0, c-a)=1$, and by Lemmas 4.7 and $4.8, \psi$ preserves layer $L_{1}$. Hence $d(0, \psi(c-a))=1$, implying $d(\phi(a), \phi(c))=1$. This proves that $\phi$ preserves distance 1 , and hence that $\phi$ is an isometry. 


\section{Concluding remarks}

The results from Sections 3 and 4 show that the concepts of $p$-isometries that are not isometries and eigenvalue collapsing are closely related but not equivalent. The existence of a $p$-isometry that is not an isometry implies eigenvalue collapsing but not vice versa. It is unfortunate that the restriction $2 p<n$ or $p=n$ is necessary in the proofs of Section 4. Although we strongly believe the following conjecture is true, we did not succeed in proving it. (However, by computing orders of automorphism groups in Sage we did not succeed in finding any $p$-isometry that is not an isometry of $H(n, q), q>2$.)

Conjecture 5.1. Every $p$-isometry of $H(n, q), q>2$, is an isometry.

We would like to end with several open problems.

- Prove Conjecture 5.1.

- Determine all triples $(q, n, p)$ for which the adjacency matrix of the distance $p$-graph of $H(n, q)$ has fewer than $n+1$ distinct eigenvalues.

- More generally, find necessary and sufficient conditions for eigenvalue collapsing to occur in a distance regular graph.

- Generalize our results on $p$-isometries to other (primitive) distance regular graphs.

\section{References}

[1] P. Abramenko, H. Van Maldeghem, Maps between buildings that preserve a given Weyl distance, Indag. Math. 15(3) (2004) 305-319.

[2] F. S. Beckman, D. A. Jr. Quarles, On isometries of Euclidean spaces, Proc. Amer. Math. Soc. 4 (1953) 810-815.

[3] A. Brouwer, A. Cohen, A. Neumaier, Distance-Regular Graphs, Springer-Verlag, Berlin, Heidelberg, 1989.

[4] A. E. Brouwer, M. A. Fiol, Distance-regular graphs where the distance $d$-graph has fewer distinct eigenvalues, Linear Algebra Appl. 480 (2015) 115-126.

[5] S. De Winter, M. Korb, Weak isometries of the Boolean cube, Discrete Math. 339(2) (2016) 877-885.

[6] E. Govaert, H. Van Maldeghem, Distance-preserving maps in generalized polygons. I. Maps on flags, Beitrage Algebra. Geom. 43(1) (2002) 89-110.

[7] E. Govaert, H. Van Maldeghem, Distance-preserving maps in generalized polygons. II. Maps on points and/or lines, Beitrage Algebra Geom. 43(2) (2002) 303-324.

[8] V. Yu. Krasin, On the weak isometries of the Boolean cube, Diskretn. Anal. Issled. Oper. Ser. 1 13(4) (2006) 26-32; translation in J. Appl. Ind. Math. 1(4) (2007) 463-467. 